\title{
Water and $\mathrm{OH}$ Maser Emission from the Planetary Nebula K3-35
}

\author{
Yolanda Gómez \\ Instituto de Astronomía, UNAM-Morelia, Apdo. Postal 3-72 (Xangari), \\ 58089 Morelia, Michoacán, México
}

Luis F. Miranda

Instituto de Astrofísica de Andalucía, CSIC, Apdo. Correos 3004, E-18080 Granada, Spain

Guillem Anglada

Instituto de Astrofísica de Andalucía, CSIC, Apdo. Correos 3004, E-18080 Granada, Spain, and Harvard-Smithsonian CfA, 60 Garden Street, Cambridge, MA 02138, USA

José M. Torrelles

Institut d'Estudis Espacials de Catalunya (IEEC/CSIC) and Instituto de Ciencias del Espacio (CSIC), Edifici Nexus, C/ Gran Capità 2-4, E-08034 Barcelona, Spain

Abstract. Water-vapour masers, typical of the envelopes in giant stars, are not expected to persist in planetary nebulae due to the ultraviolet radiation of the remnant star that progressively destroys the molecules. Recently, we have reported the first unambiguous detection of water maser emission in a planetary nebula, K 3-35 (Miranda et al. 2001). The water masers in K3-35 were detected at the center of the nebula, along the minor axis, at a radius of $\sim 85 \mathrm{AU}$ and also at the surprisingly large distance of $5000 \mathrm{AU}$ from the star, at the tips of the bipolar lobes. The existence of these water molecules is puzzling, and probably we are observing the very moment of transformation of a giant star into a planetary nebula. Miranda et al. (2001) also report the presence of polarization in the $\mathrm{OH}$ $1665 \mathrm{MHz}$ masers, which are distributed towards the central star in a torus-like structure. Here we review the main results on this source.

\section{Introduction}

$\mathrm{K}$ 3-35 is an emission nebula that has been studied at optical, radio and infrared wavelengths. At radio and optical wavelengths it is characterized by an S-shaped emission morphology with a clear point-symmetric structure that attracted the attention of several authors (Aaquist \& Kwok 1989; Aaquist 1993; Miranda et al. 2000; 2001). Point-symmetric morphologies have been observed in many planetary nebulae (Guerrero et al. 1999; López 2002). There was 
some controversy in the past about the nature of K3-35 (YSO vs. PN), since it appears projected towards the molecular cloud L 755. However, the currently available data (Miranda et al. 2000; 2001, and references therein) strongly support the classification of K3-35 as a PN. A summary of the observational data that strongly supports the PN classification are: the presence of strong $\mathrm{He}^{+}$ emission at $4686 \AA$, strong [NII] 6548 and $6583 \AA$ emission lines $\left([\mathrm{NII}] / \mathrm{H}_{\alpha} \simeq 5\right)$, the presence of $[\mathrm{ArIV}]$ and $[\mathrm{OIII}]$ emission lines $\left([\mathrm{OIII}] / \mathrm{H}_{\beta} \simeq 30\right)$, the location of K3-35 in the region occupied by OH/IR stars and PNe in the IRAS two-colour diagram, the OH maser emission from K3-35 is type II (typical in evolved stars). The sensitive observations of Dayal \& Bieging (1996) reveal that the CO lines present two velocity components, a narrow component associated with the L755 cloud and a broader component apparently associated with K3-35.

Engels et al. (1985), through single-dish observations, detected water maser emission towards a position close to that of K3-35, but with their angular resolution $\left(\simeq 40^{\prime \prime}\right)$ it was not possible to establish whether this emission originates from K3-35 itself or from a YSO associated with the background molecular cloud L755. Also, the nature of K3-35 itself was unclear at that time, and an interpretation in terms of an HII region was favored. Water masers, typical of giant envelopes, were not expected in a PN because water molecules are rapidly destroyed when photoionization begins (Lewis 1989; Gómez, et al. 1990). In order to investigate the association of the water masers with K3-35, we carried out high angular resolution observations using the Very Large Array (VLA) of $\mathrm{NRAO}^{8}$ in the A configuration. A detailed description of the observations and the main results obtained have been presented in Miranda et al. (2001).

\section{Results and Discussion}

We detect towards K 3-35 four active water maser regions located at very significant positions (see Figure 1; left, Table 1). A group of masers are located towards the core of the nebula, tracing the inner regions of a disk-like structure, while the rest are at the tips of the bipolar lobes, at a considerable distance of $\sim 5000$ UA from the central star (assuming a distance of $5 \mathrm{kpc}$ to the nebula; Zhang 1995). This is the first object in the sky where water masers are detected at the tips of the precessing jets, which we believe are involved in the excitation mechanism. The water masers appear at an LSR velocity range from 20 to 40 $\mathrm{km} \mathrm{s}^{-1}$, and the strongest maser feature is located at the center of the nebula.

The $\mathrm{OH} 1665$ and $1667 \mathrm{MHz}$ masers were detected towards the bright core of K3-35 (see Figure 1; right, Table 2) confirming the association with the nebula. The $1665 \mathrm{MHz}$ masers are distributed in a band perpendicular to the outflow with a radius $\simeq 630 \mathrm{AU}$, suggesting a torus-like structure. The $1667 \mathrm{MHz}$ masers are located in two main groups, each displaced $\simeq 800 \mathrm{AU}$ from the center, oriented along the outflow direction. Strong levels of circular polarization are present in the $1665 \mathrm{MHz}$ line, suggesting the presence of a magnetic field of order of $\mathrm{mG}$. It is the first time that a magnetic field is found in a torus-like structure, as required by some theoretical models (Rozyczka \& Franco 1996; García-Segura 1997).

\footnotetext{
${ }^{8}$ The National Radio Astronomy Observatory is a facility of the National Science Foundation operated under cooperative agreement by Associated Universities, Inc.
} 

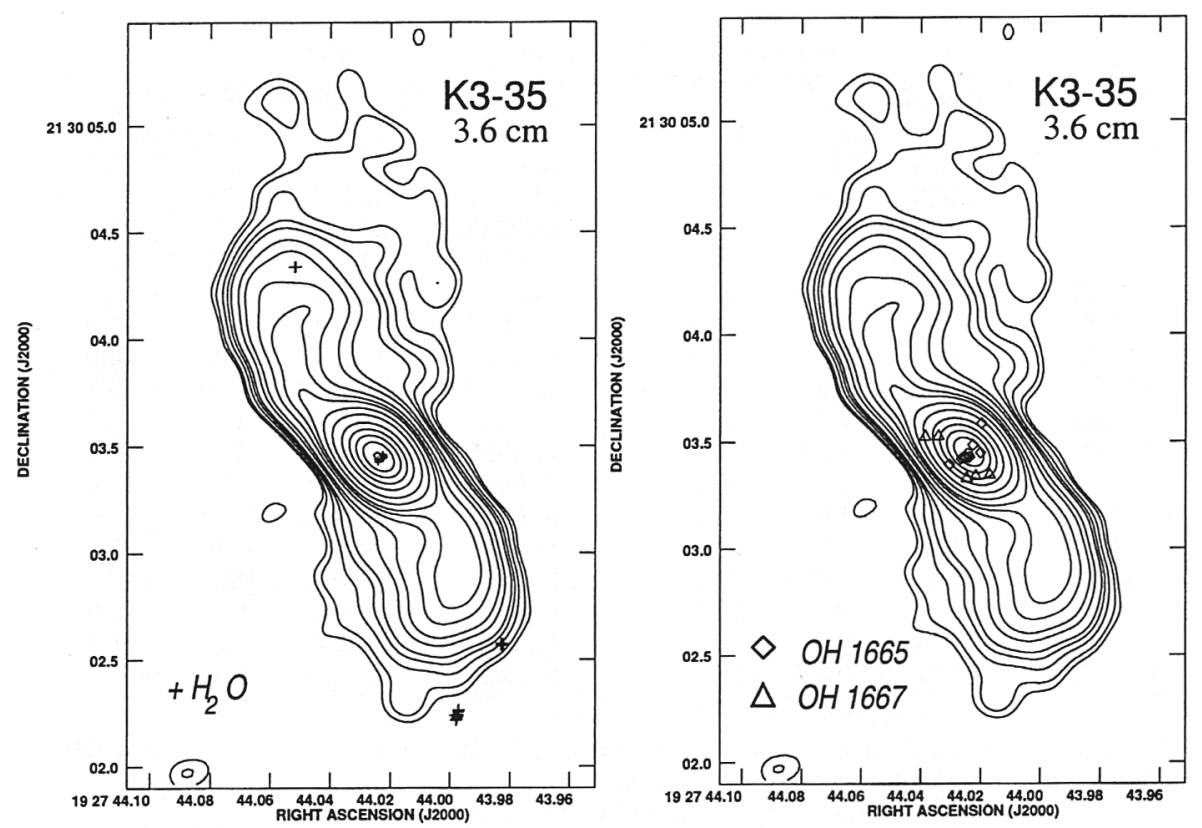

Figure 1. VLA 3.6-cm continuum contour map of K3-35 (beam size is 0 "'2). Positions of the $\mathrm{H}_{2} \mathrm{O}$ (left; crosses), $\mathrm{OH} 1665$ (right; diamonds), and $\mathrm{OH} 1667$ (right; triangles) maser spots are indicated. The LSR velocities and fluxes of the maser spots are given in Tables 1 and 2. The contours of the 3.6 continuum emission are $-3,3,4,6,8,10,15,20,30,40,50,70,100,150$, $200,250,300$, and 350 times $0.036 \mathrm{mJy} \mathrm{beam}^{-1}$, the rms noise of the map (Miranda et al. 2001).

Acknowledgments. YG acknowledges the support from DGAPA, UNAM, and CONACyT, México. LFM, GA, and JMT acknowledge partial support from MCYT grant PB98-0670-C02 and from Junta de Andalucía, Spain. GA also acknowledges support from MEC, Spain and from SAO, USA.

\section{References}

Aaquist, O.B. 1993, A\&A, 267, 260

Aaquist, O.B., \& Kwok, S. 1989, A\&A, 222, 227

Dayal, A., \& Bieging, J.H. 1996, ApJ, 472, 703

Engels, D. et al. 1985, A\&A, 148, 344

García-Segura, G. 1997, ApJ, 489, L189

Gómez, Y., Moran, J.M., \& Rodríguez, L.F. 1990, RMAA, 20, 55

Guerrero, M.A., Vázquez, R., \& López, J.A. 1998, ApJ, 117, 967

Lewis, B.M. 1989, ApJ, 338, 234

López, J.A. 2002, Proceedings of IAU Symp 209, in press.

Miranda, L.F. et al. 2000, MNRAS, 311, 748

Miranda, L.F., Gómez, Y., Anglada, G., \& Torrelles, J.M. 2001, Nature, 414, 284 
Table 1. Parameters of the $\mathrm{H}_{2} \mathrm{O}$ Masers in $\mathrm{K} \mathrm{3-35^{a }}$

\begin{tabular}{ccccccccc}
\hline \hline $\begin{array}{c}\alpha(\mathrm{J} 2000)^{b} \\
(\mathrm{~s})\end{array}$ & $\begin{array}{c}\delta(\mathrm{J} 2000)^{b} \\
\left({ }^{\prime \prime}\right)\end{array}$ & $\begin{array}{c}S_{\nu} \\
(\mathrm{mJy})\end{array}$ & $\begin{array}{c}\mathrm{V}_{L S R}^{c} \\
\left(\mathrm{~km} \mathrm{~s}^{-1}\right)\end{array}$ & & $\begin{array}{c}\alpha(\mathrm{J} 2000)^{b} \\
(\mathrm{~s})\end{array}$ & $\begin{array}{c}\delta(\mathrm{J} 2000)^{b} \\
\left({ }^{\prime \prime}\right)\end{array}$ & $\begin{array}{c}S_{\nu} \\
(\mathrm{mJy})\end{array}$ & $\begin{array}{c}\mathrm{V}_{L S R}^{c} \\
\left(\mathrm{~km} \mathrm{~s}^{-1}\right)\end{array}$ \\
\hline 43.9821 & 02.573 & 21 & 36.5 & & 44.0517 & 04.339 & 64 & 25.3 \\
43.9825 & 02.573 & 70 & 35.8 & & 43.9971 & 02.266 & 121 & 24.6 \\
43.9826 & 02.574 & 75 & 35.1 & & 44.0243 & 03.439 & 23 & 24.6 \\
43.9828 & 02.575 & 26 & 34.5 & & 44.0519 & 04.338 & 298 & 24.6 \\
43.9825 & 02.573 & 33 & 32.5 & & 43.9970 & 02.264 & 96 & 24.0 \\
43.9827 & 02.570 & 56 & 31.9 & & 44.0242 & 03.428 & 61 & 24.0 \\
43.9830 & 02.567 & 32 & 31.2 & & 44.0520 & 04.337 & 795 & 24.0 \\
43.9977 & 02.224 & 16 & 31.2 & & 43.9970 & 02.260 & 57 & 23.3 \\
43.9980 & 02.217 & 33 & 30.5 & & 44.0225 & 03.446 & 218 & 23.3 \\
43.9978 & 02.222 & 44 & 29.9 & & 44.0521 & 04.337 & 1196 & 23.3 \\
43.9975 & 02.233 & 55 & 29.2 & & 43.9983 & 02.239 & 15 & 22.6 \\
43.9975 & 02.234 & 59 & 28.6 & & 44.0223 & 03.452 & 1010 & 22.6 \\
43.9975 & 02.238 & 67 & 27.9 & & 44.0521 & 04.337 & 822 & 22.6 \\
43.9973 & 02.245 & 107 & 27.2 & & 44.0224 & 03.453 & 1572 & 22.0 \\
43.9973 & 02.255 & 163 & 26.6 & & 44.0521 & 04.336 & 175 & 22.0 \\
43.9972 & 02.264 & 221 & 25.9 & & 44.0225 & 03.456 & 945 & 21.3 \\
43.9971 & 02.267 & 193 & 25.3 & & 44.0226 & 03.463 & 201 & 20.7 \\
\hline
\end{tabular}

a As observed on September 6, 1999.

${ }^{b}$ Right ascension is $19^{h} 27^{m}$ and declination is $+21^{\circ} 30^{\prime}$. Relative positional errors are 0 ! $^{\prime} 001$. Uncertainty in the absolute coordinates is $0 . \prime 05$.

$c$ Velocity at the center of the channel (channel width is $0.6 \mathrm{~km} \mathrm{~s}^{-1}$ ).

Table 2. Parameters of the $\mathrm{OH}$ masers in $\mathrm{K} 3-35^{a}$

\begin{tabular}{|c|c|c|c|c|c|c|c|}
\hline \multicolumn{4}{|c|}{$\mathrm{OH} 1665$} & \multicolumn{4}{|c|}{$\mathrm{OH} 1667$} \\
\hline $\begin{array}{c}\alpha(\mathrm{J} 2000)^{b} \\
(\mathrm{~s})\end{array}$ & $\begin{array}{c}\delta(\mathrm{J} 2000)^{b} \\
\left({ }^{\prime \prime}\right)\end{array}$ & $\begin{array}{c}S_{\nu} \\
(\mathrm{mJy})\end{array}$ & $\begin{array}{c}\mathrm{V}_{L S R}^{c} \\
\left(\mathrm{~km} \mathrm{~s}^{-1}\right)\end{array}$ & $\begin{array}{c}\alpha(\mathrm{J} 2000)^{b} \\
(\mathrm{~s})\end{array}$ & $\begin{array}{c}\delta(\mathrm{J} 2000)^{b} \\
\left({ }^{\prime \prime}\right)\end{array}$ & $\begin{array}{c}S_{\nu} \\
(\mathrm{mJy})\end{array}$ & $\begin{array}{c}\mathrm{V}_{L S R}^{c} \\
\left(\mathrm{~km} \mathrm{~s}^{-1}\right)\end{array}$ \\
\hline 44.0226 & 03.485 & 14 & 22.2 & 44.0169 & 03.343 & 16 & 9.0 \\
\hline 44.0197 & 03.584 & 13 & 21.1 & 44.0218 & 03.339 & 38 & 7.9 \\
\hline 44.0201 & 03.449 & 40 & 20.0 & 44.0247 & 03.329 & 30 & 6.8 \\
\hline 44.0239 & 03.428 & 121 & 18.9 & 44.0386 & 03.523 & 13 & -1.9 \\
\hline 44.0249 & 03.427 & 144 & 17.8 & 44.0342 & 03.524 & 17 & -3.0 \\
\hline 44.0257 & 03.429 & 98 & 16.7 & & & & \\
\hline 44.0256 & 03.427 & 107 & 15.6 & & & & \\
\hline 44.0265 & 03.421 & 106 & 14.1 & & & & \\
\hline 44.0306 & 03.393 & 39 & 13.4 & & & & \\
\hline
\end{tabular}

${ }^{a}$ As observed on September 6, 1999.

$b$ Right ascension is $19^{h} 27^{m}$ and declination is $+21^{\circ} 30^{\prime}$. Relative positional errors are $0 !^{\prime \prime} 01$ for the $1665 \mathrm{MHz}$ data and 0 .' 05 for the 1667 data. Uncertainty in the absolute coordinates is $0 . " 05$.

$c$ Velocity at the center of the channel (channel width is $1.1 \mathrm{~km} \mathrm{~s}^{-1}$ ).

Rozyczka, M., \& Franco, J. 1996, ApJ, 469, L127

Zhang, C.Y. 1995, ApJS, 98, 659 